\title{
Red de Tecnoparques del Servicio Nacional de Aprendizaje - SENA - Colombia. Una mirada desde la línea de ingeniería y diseño nodo Bogotá
}

\author{
Adela Marcela Triana Arevalo ${ }^{a}$, John Eduard Gómez Bohórquez ${ }^{\text {b }}$ y Juan Sebastian \\ Avila Foreroc \\ a Diseñadora Industrial, Gestora Tecnoparque SENA Nodo Bogotá, adelmar87@misena.edu.co, \\ bIngeniero Aeronáutico, Gestor Tecnoparque SENA Nodo Bogotá, jeduard.gomez@misena.edu.co, \\ ${ }^{\mathrm{c}} \mathrm{PhD}(\mathrm{c})$, Profesor de Diseño Industrial Universidad El Bosque de Bogotá, \\ avilajuan@unbosque.edu.co.
}

\begin{abstract}
The National Service of Learning (SENA) is a Colombian public institution that offers technical training programs for human resources for the labor market. Performs business, community and technological development activities.

The SENA Technoparks are a technological innovation program aimed at all Colombians, which acts as an accelerator for the development of $R \& D \& I$ projects materialized in functional prototypes in four technological lines that promote technology-based entrepreneurship: Electronics and Telecommunications, Virtual Technologies, Biotechnology, Nanotechnology and Engineering and Design.

Specifically, for the Engineering and Design line, we present their services and results in the following areas: Analysis and simulation, industrial design, product and process engineering, design and materials for manufacturing and assembly, digital product manufacturing, reverse engineering. Illustrating the developments through projects, which have the potential to become companies with technological impact or in outstanding opportunities for employment and sales of products or services.
\end{abstract}

Keywords: Innovation, $R \quad \& \quad D$, FabLab, Technical education, Entrepreneurship.

\section{Resumen}

El Servicio Nacional de Aprendizaje SENA (Sena.edu.co, 2018) es una institución pública colombiana que ofrece programas de formación complementaria y titulada. Enfatiza su misión en la capacitación técnica del recurso humano; formando personas para vincularlas al mercado laboral y realiza actividades de desarrollo empresarial, comunitario y tecnológico. 
Los Tecnoparques SENA, (Tecnoparque.sena.edu.co, 2018) son un programa de innovación tecnológica dirigida a todos los Colombianos, que actúa como acelerador para el desarrollo de proyectos de $I+D+i$ materializados en prototipos funcionales en cuatro líneas tecnológicas que promueva el emprendimiento de base tecnológica: Electrónica y Telecomunicaciones, Tecnologías Virtuales, Biotecnología, Nanotecnología e Ingeniería y Diseño.

Específicamente, para la línea de Ingeniería y Diseño queremos dar a conocer sus servicios y resultados en las siguientes áreas: planeación, análisis y simulación, diseño industrial, ingeniería de productos y procesos, diseño y materiales para la manufactura y ensamble, fabricación digital de productos, ingeniería inversa. Ilustrando los desarrollos a través de algunos proyectos, que tienen potencial de convertirse en empresas de impacto tecnológico o en oportunidades destacadas de vinculación laboral, o venta de productos o servicios.

Palabras clave: Innovación, I + D, FabLab, Educación técnica, Emprendimiento.

\section{Introducción}

El SENA fue fundado en 1957, cuenta con varias sedes en Bogotá y en los 32 departamentos de Colombia, el enfoque principal del SENA durante sus primeros años fue brindar instrucción técnica a los trabajadores y empleados de la industria, el comercio y el sector agropecuario. Sin embargo, en la actualidad atiende la demanda de sectores como el financiero, tecnológico y de la salud. Lo anterior lo ha llevado a convertirse en una de las mejores alternativas de instrucción para colombianos de escasos recursos, así como trabajadores y empresarios. La oferta regular para formación profesional: técnicos, tecnólogos y trabajadores especializados incluye más de 522 programas. A lo largo de sus más de 50 años el SENA se ha destacado por ser uno de los más importantes centros de educación técnica de América Latina. (Lineabase.sena.edu.co, 2018)

El programa Red TecnoParque Colombia, es una iniciativa de innovación tecnológico del SENA adscrito a la Dirección de Formación Profesional - Grupo gestión estratégica de la investigación, desarrollo tecnológico e innovación SENNOVA, (Sena.edu.co, 2018), para el desarrollo integral del talento humano, que, con ambientes de aprendizaje especializados para la apropiación de innovación y tecnología, complementa la formación ofrecida por el SENA e integra actores adicionales de los sectores público, privado y educativo.

El SENA, desde el programa Red Tecnoparque Colombia cuenta con cuatro líneas de investigación y apoyo a los empresarios. La línea de ingeniería y diseño, el cual tiene como función: prestar sus servicios en las siguientes áreas: Análisis y simulación, diseño industrial, ingeniería de productos y procesos, diseño y materiales para la manufactura y ensamble, fabricación digital de productos e ingeniería inversa. De esta manera se ha promovido y potencializando la creación de negocios a partir del uso de las herramientas del diseño industrial y la ingeniería; a través de la asesoría, acompañamiento a iniciativas orientadas hacia la optimización, desarrollo de planes de acción para la diversificación y 
diferenciación de la oferta del sector productivo nacional, impulsando la innovación y el emprendimiento de los aprendices.

\section{Objetivos:}

Socializar las actividades y gestión llevada a cabo durante el funcionamiento y operación de la línea ingeniería y diseño de TecnoParque nodo Bogotá, evidenciando los aciertos y dificultades de los proyectos asesorados en el periodo 2015-2017.

\subsection{Objetivos específicos:}

1. Dar a conocer la experiencia en el desarrollo de proyectos innovadores de base tecnológica desde el nodo Tecnoparque de Bogotá, que han contribuido al crecimiento económico y la competitividad de pequeñas empresas en Colombia.

2. Ilustrar algunos factores de articulación entre gobierno, empresa y academia para el desarrollo de acciones conjuntas enfocadas hacia la innovación.

3. Evidenciar los escenarios donde los aprendices y futuros emprendedores colombianos, desarrollan proyectos de base tecnológica con alto potencial innovador, pudiendo materializar prototipos funcionales y productos tecnológicos con el apoyo de una entidad pública.

\section{Innovación educativa a partir de proyectos de base tecnológica:}

La estrategia de la línea de ingeniería y diseño está establecida en diferentes ejes, el primero de ellos es la diferenciación en la aplicación de conceptos de diseño en el desarrollo y consecución de los proyectos de base tecnológica, el segundo la integración de planes de acción dependiendo del tipo del proyecto vinculado al nodo, siendo la personalización en el servicio a cada uno de los aprendices uno de los factores fuertes del programa. El tercero la planificación como proceso de definición sistémica en los lineamientos para la toma de decisiones, la asignación y distribución de los recursos y la consecución de los objetivos principales del proyecto vinculado al nodo.

Para las instituciones de formación de profesionales del país en áreas de diseño, salud, ingeniería diseño automotriz entre otras, esta iniciativa permite a los emprendedores, pymes y público en general contar con la infraestructura de la línea para ayudar a apalancar el desarrollo del país a través de la especialización en línea de desarrollo tecnológico (LDT) con prototipos y productos mínimos viables (MVP, del inglés Minimum Viable Product). (Producto viable mínimo, 2017).

Con estos prototipos funcionales los aprendices representan sus ideas de diseño antes de obtener un producto final. En algunas industrias o empresas la palabra prototipo se utiliza para versiones de productos próximos a lanzar a un mercado como el producto final. (Avila Forero, J. S. 2016). Con esta estratègia se ha ido articulando y haciendo transferencia de conocimiento y de tecnología para las redes de trabajo de acuerdo con las habilidades y especialización de cada nodo según las habilidades de cada uno de los estudiantes que se inscriben al programa. 
Dentro de los servicios que tiene la línea de ingeniería y diseño está el atender a la población objetivo, como aprendices, técnicos y tecnólogos que se encuentran en los centros de formación del SENA en cualquier programa de formación, al igual que instructores SENA, grupos de investigación, empresas, entidades de educación y público en general. La asesoría de cada proyecto es un acompañamiento técnico y personalizado para el desarrollo del proyecto. El acompañamiento se hace por parte de un equipo interdisciplinario de profesionales con experiencia y formación académica para el análisis, planeación, diseño, ingeniería, desarrollo, pruebas y simulación de proyectos y prototipos tanto virtuales como materiales.

\subsection{Trabajo en nodos como estrategia para optimizar recursos:}

La operación del programa de la Red de Tecnoparques de Colombia se desarrolla a través de nodos, entendidos como unidades adscritas a un Centro de Formación o Dirección Regional de la jurisdicción en que se encuentre ubicado en el país. El recurso humano de la planta de personal y los contratistas que requieran los nodos para su funcionamiento, están a cargo del Centro de Formación o Regional al cual esté adscrito. Desde 2015 se cuenta a nivel nacional con quince (15) nodos: dos en Antioquia (Rionegro y Medellín), uno en Tolima (La Granja), uno en Caldas (Manizales), dos en Santander (Bucaramanga y Socorro), uno en Cundinamarca (Cazucá), uno en Valle (Cali), uno en Cesar (Valledupar), tres en Huila (Pitalito, Neiva y La Angostura), uno en Risaralda (Pereira), uno en Norte de Santander (Ocaña) y uno en Bogotá. Estos nodos cuentan con infraestructuras similares donde se comparten desarrollos administrativos, conocimientos técnicos, métodos de trabajo y filosofías misionales para apoyar a los estudiantes, aprendices y emprendedores de Colombia en todas las regiones del país. (Tecnoparque.sena.edu.co, 2018)

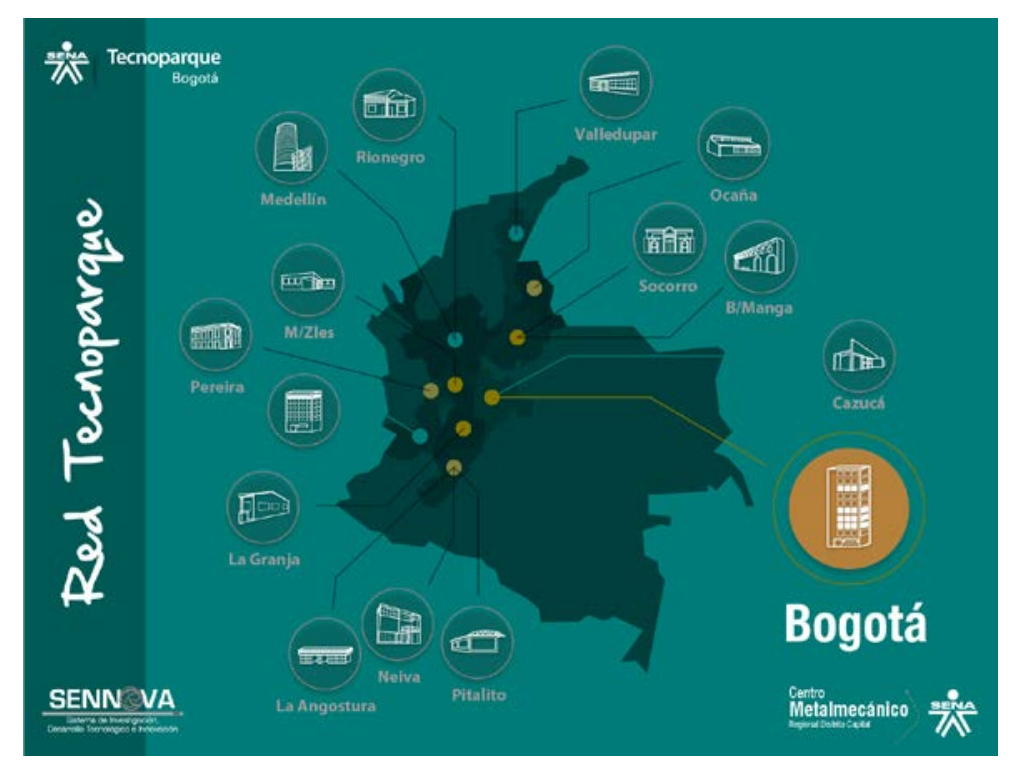

Fig. 1. Ubicación de Nodos Tecnoparque en la Geografía Colombiana. Autor Corporativo. 2016 
A través del programa de la red Tecnoparque SENA, se busca conectar el sector productivo con el talento formado en el SENA a través de procesos de innovación abierta, para desarrollar de forma colaborativa proyectos de I+D+i, compartiendo los recursos, el talento humano, información estratégica y diferentes conocimientos entre los quince nodos. De esta forma se apropia el conocimiento interno y externo existente, generando ventajas competitivas en el mercado para los actores que se forman en estos centros, co-ejecutando Proyectos de Base Tecnológica (PBT) y acciones internodos que apuntan al fortalecimiento de trabajo en red.

\subsection{Eventos de Divulgación Tecnológica (EDT):}

Como parte de las estrategias desarrolladas en la Red Tecnoparque, se ha propuesto una forma de compartir avances tecnológicos, formas de hacer y transferir conocimientos denominados EDT. Un EDT es una socialización grupal sobre temas puntuales de actualidad tecnológica dictados por especialistas, generalmente de forma presencial a modo de conferencia o taller, estos eventos ayudan a aprendices e instructores a conocer y apropiarse de nuevas competencias técnicas. Los EDT se dividen en dos categorías fundamentalmente:

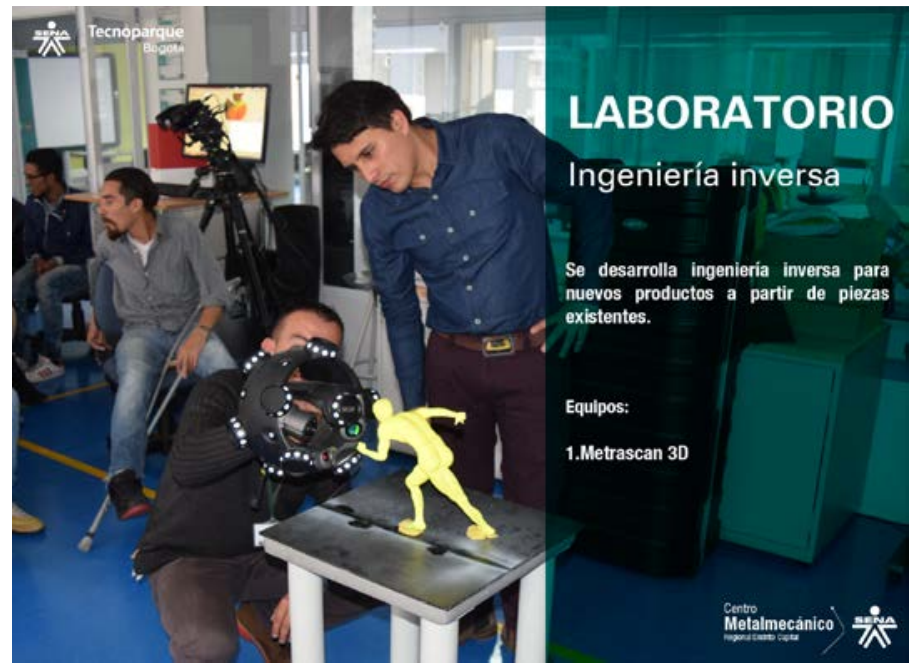

Fig. 2. EDT Procesos de Ingeniería Inversa y Escaneo 3D. Tecnoparque Nodo Bogotá. 2016

\subsubsection{Eventos de conocimiento y tecnología:}

Cuyo objetivo es poner al alcance de los usuarios de la Red Tecnoparque como empresas, academia y comunidad SENA, nuevas tecnologías, técnicas de actualidad, lecciones aprendidas, experiencias en convocatorias de Innovación, entre otros. La información de los expositores en estos eventos alimenta una base de datos de la Red de aliados y expertos que permite direccionar futuros proyectos con los recursos idóneos, permitiendo optimizar recursos y agilizar procesos. 


\subsubsection{Talleres de innovación:}

Son sesiones dinámicas que ponen en práctica los principios de la metodología de Design Thinking (Brown, T. 2016), para la generación colaborativa de ideas innovadoras y pasos adicionales para la solución a las problemáticas o necesidades de las empresas participantes en convocatorias de innovación abierta de la Red Tecnoparque.

\section{Metodología “NoteBooks” de desarrollo de los Nodos Tecnoparque:}

La metodología de desarrollo de proyectos de la Red Tecnoparque, consiste en once (11) actividades a ser realizadas en equipo, mediante un proceso interactivo con la plataforma de gestión de información GDrive de Google, bajo unos formatos estandarizados, que permiten regular la información de forma eficaz desde cualquier computador conectado a internet y en tiempo real (Canchica de Medina, Marbelys Elizabeth. 2016). Si los resultados obtenidos tras el desarrollo de una actividad o módulo no son satisfactorios, el equipo debe regresar a la actividad anterior con el fin de identificar posibles oportunidades de mejora y realizar los ajustes necesarios, todo en tiempo real.

Las actividades para realizar un proyecto en la Red son las siguientes:

Inspiración:

1. Observación.

2. Vigilancia tecnológica.

3. Prospectiva.

Ideación:

4. Concepto.

5. Sostenibilidad.

6. Modelo de Negocio.

7. Validación.

Prototipado:

8. Preparación.

9. Creación.

10. Verificación y testeo.

11. Retroalimentación. 


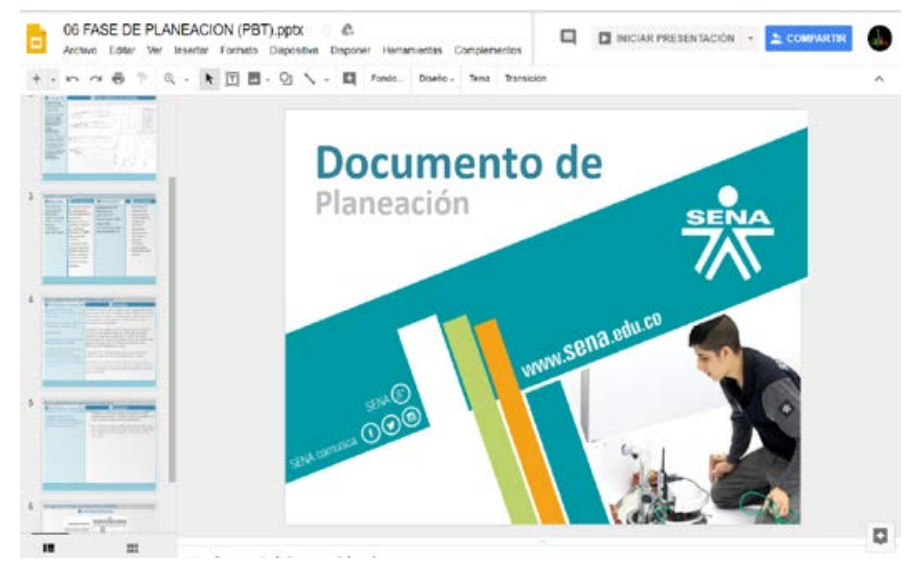

Fig. 3. Captura de pantalla, documento de trabajo en la plataforma de GDrive de Google. Autor Corporativo. 2016

Para facilitar la apropiación de la metodología, existe una guía de trabajo, que aborda desde cada una de las fases, etapas y actividades que la componen y que son necesarias para la ejecución de un proyecto desarrollado en un nodo Tecnoparque. De igual manera cada uno de los lectores podrá apoyarse en un Book de GDrive, con la información, descripción y documentación necesaria para desarrollar cada una de las fases, etapas y actividades de la metodología.

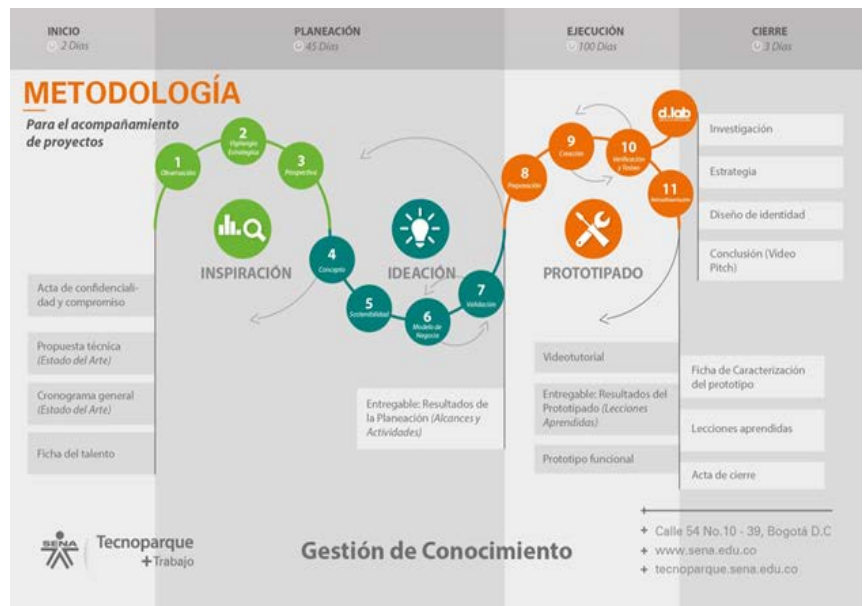

Fig. 4. Metodología para el acompañamiento de proyectos. Tecnoparque. Autor Corporativo. 2016

\section{Resultados destacados:}

\subsection{Simulador de entrenamiento por laparoscopia:}

El desarrollo y masificación de las tecnologías de diseño y fabricación digital en las últimas dos décadas, ha permitido que un número creciente de emprendedores comiencen a 
formular proyectos de base tecnológica en diferentes ámbitos y sectores económicos. La impresión aditiva se ha convertido en un factor de alto impacto y en la tecnología más representativa de ésta nueva forma de materializar ideas. En el campo de las áreas de la salud, específicamente en el diseño y la fabricación de simuladores se ha encontrado un nicho de desarrollo importante de aplicación. Como resultado a estas dinámicas, se desarrolló un prototipo de simulador de entrenamiento para operaciones laparoscópicas en asocio con investigadores universitarios en cabeza del profesor de diseño industrial Juan Sebastián Ávila de la Universidad El Bosque de Bogotá, una universidad privada enfocada en áreas de la salud y gestores de proyecto del Tecnoparque del SENA, bajo la convocatoria de apoyo a proyectos de SENNOVA 2016. Este proyectos es un claro ejemplo de las dinámicas y resultados del trabajo coordinado y en red entre diferentes instituciones, donde cada uno de los integrantes tiene un rol determinado, que fue esencial para el desarrollo exitoso del proyecto, por una parte, desde el Tecnoparque se ofrece todo el soporte y respaldo técnico y tecnológico para materializar ideas, y por otra parte la Universidad El Bosque aporta el conocimiento científico y los escenarios de experimentación y comprobación real del proyecto, siendo un proyecto significativo al unir dos grandes áreas del conocimiento como lo es la medicina y la ingeniería - diseño, vinculando no solamente a profesionales, sino que también a estudiantes en formación. Por otra, parte, éste proyecto también es un buen ejemplo del trabajo coordinado entre una entidad de carácter público y una entidad de carácter privado, que fueron capaces de sacar adelante un proyecto conjunto, superando las diferencias de gestión y manejo administrativo.

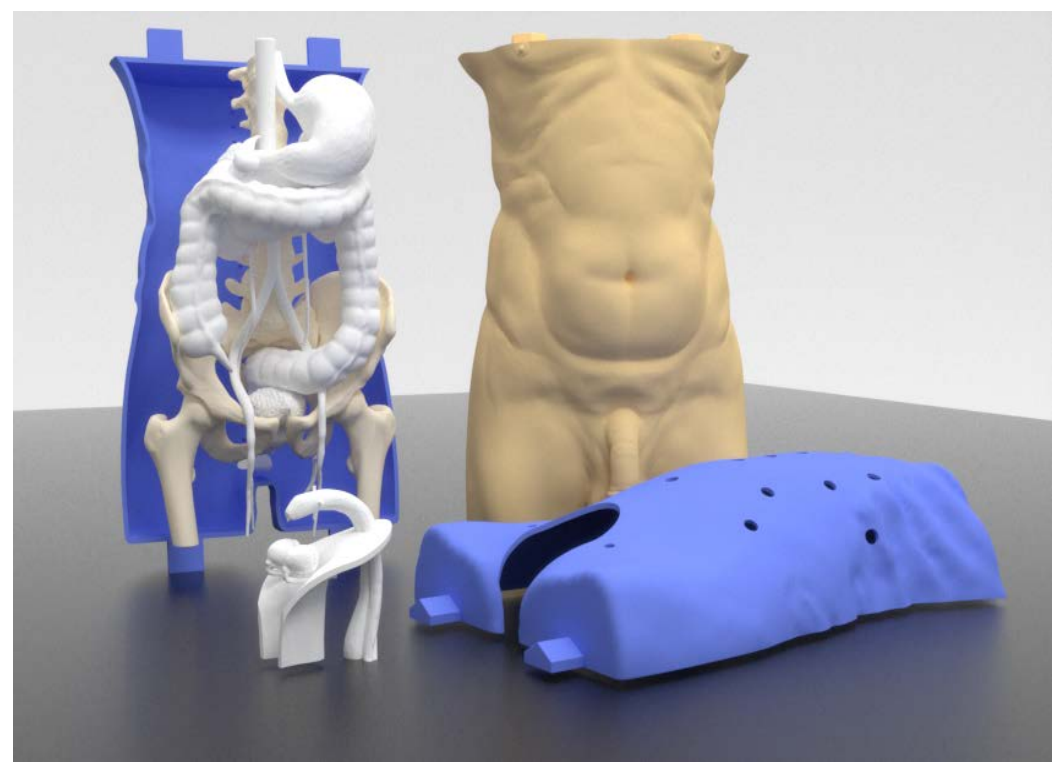

Fig.5. Modelado 3D, Proyecto Simulador de entrenamiento para cirugías por laparoscopia. 2016

\subsection{Pintack:}


Este proyecto fue realizado por el aprendiz SENA Iván Hernández en 2016, quien desarrolló una herramienta de mano resistente cuya principal función es la de sostener puntillas y grapas al momento de ser clavadas manteniendo los pulgares y otros apéndices de forma segura. PinTack tiene un cómodo agarre de goma fijado en torno a una superficie sólida duradera que alberga un sistema mecánico en el extremo, capaz de sostener cualquier elemento de fijación, especialmente puntillas de 1/2" hasta 1/1/2" mediante un paquete magnético y tiene una capacidad de agarre de hasta 5 milímetros de diámetro, posee un sistema de imanes de alta potencia que permiten adherirse a la herramienta de trabajo, facilitando así su almacenaje.

Este proyecto se asesoró desde la línea de ingeniería y diseño por el Ing. John Eduard Gómez basado en la metodología para el desarrollo de proyectos, haciendo énfasis en el ciclo de vida del producto, apoyado en la lógica de diseño de producto, factores ergonómicos, secuencias de uso, materiales, mecanismos y prototipado final del producto. Este proyecto fue desarrollado en coordinación con diferentes nodos del Tecnoparque, que aportaron desde diferentes perspectivas, como el mercadeo, la ingeniería, el diseño, comunicación, entre otros. Cabe resaltar que este dispositivo se encuentra de proceso de otorgación de patente en modelo de utilidad por la Superintendencia de Industria y Comercio de Colombia, demostrando que sí es posible realizar proyectos completos, desde su fase de formulación hasta una patente y posterior comercialización en el mercado.

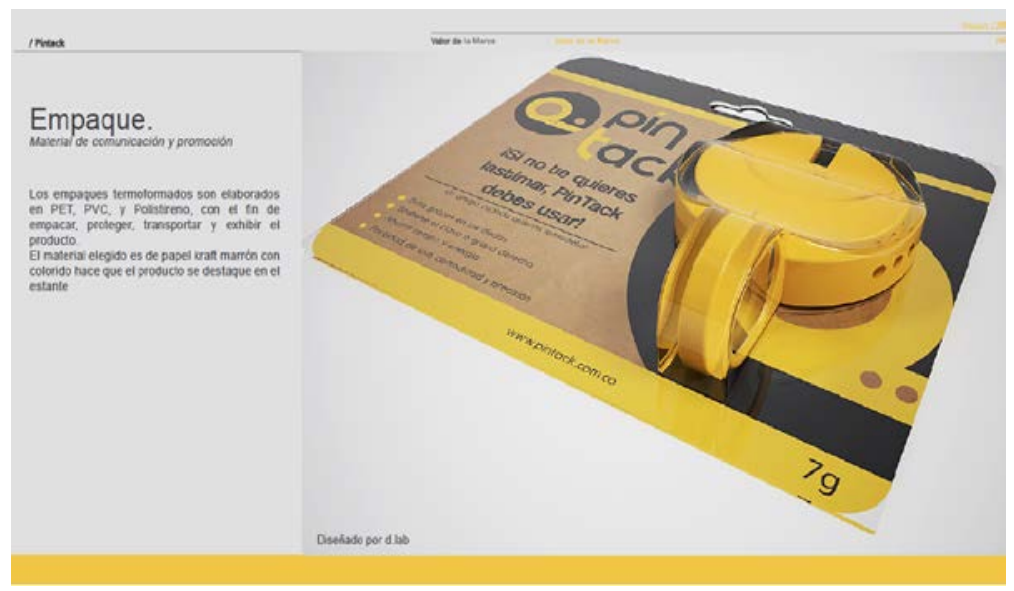

Fig. 6. Desarrollo 3D prototipo pintack-Tecnoparque. Autor Corporativo. 2016

\subsection{Patín para rehabilitación de extremidades superiores e inferiores:}

Este proyecto fue realizado por el talento Luís Alfonso Díaz, quien desarrolló varios prototipos del patín para terapias de rehabilitación, dirigido a personas con fracturas o lesiones en las extremidades superiores e inferiores. Dentro del nodo Bogotá, en la línea de Ingeniería y diseño se realizó el proceso de acompañamiento para el desarrollo de la idea, en un trabajo conjunto con aprendices SENA del curso de tecnología en diseño y desarrollo de producto industriales (Tecnología en Gestión de la Producción Industrial. 2018). que aportaron al desarrollo del diseño CAD del prototipo, en una primera fase de diseño mecánico se contó con la participación de Leonardo Zuluaga, ingeniero mecánico y dinamizador del tecnoparque, asesorando los sistemas de amortiguación, sistema de frenado 
y sistema de movimiento esto con el fin de generar un control, dominio y uso del prototipo con el usuario, para una segunda fase de desarrollo se profundizó en el factor ergonómico y estético para reflejar una mejor comunicación en la interacción del usuario con el objeto, utilizando sistemas de fabricación digital y de impresión 3D al tiempo se hizo una articulación con la Escuela Colombiana de Rehabilitación para la validación de los prototipos con expertos en ergonomía. Este proyecto es valioso en el sentido que muestra el trabajo coordinado de diferentes profesionales y entidades tanto públicas como privadas para alcanzar un bien común, articulado por conceptos de diseño, ingeniería y proyectos de base tecnológica.
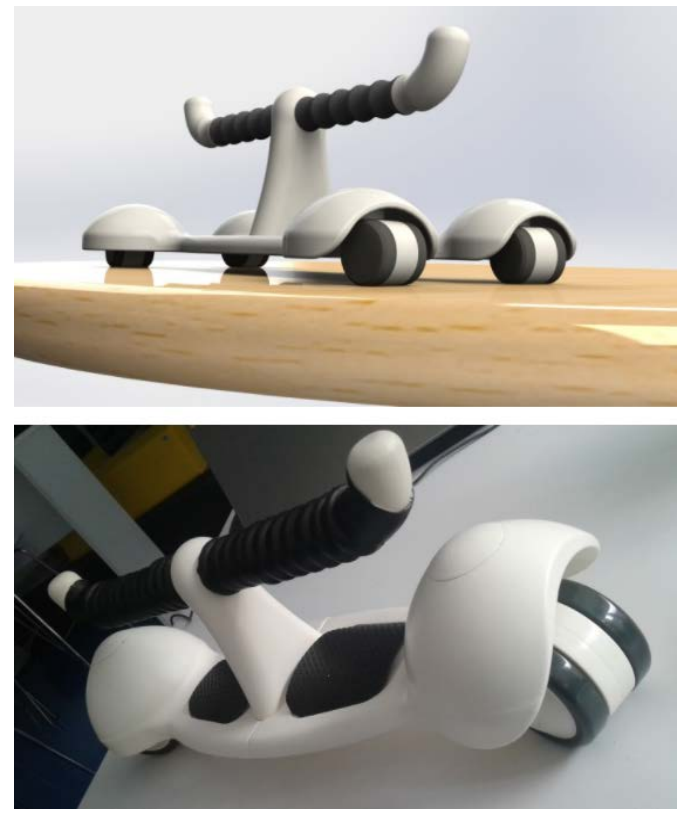

Fig. 7. Diseño modelado 3D y prototipo, proyecto patín para rehabilitación de extremidades superiores $e$ inferiores . Tecnoparque Nodo Bogotá. 2017.

\section{Conclusiones:}

La línea de diseño e ingeniería del Tecnoparque nodo de Bogotá, cuenta con una infraestructura de laboratorios y oficinas de 350 mts2 ubicados en Chapinero, una zona privilegiada por ser una de las zonas de mayor afluencia de jóvenes en el centro de la capital de Colombia. El espacio está dividido en Zona de laboratorio, Taller de construcción de prototipos, Laboratorio fabricación digital, Ambiente de asesoría y trabajo co-working. En el laboratorio de fabricación digital se cuenta con 3 impresoras 3D de tecnología FDM, un escáner 3D, una máquina universal de ensayos, una cortadora láser y una termoformadora, asimismo en el área de asesoría contamos con ordenadores de gran capacidad de procesamiento, que cuentan con programas especializados en el área de ingeniería y diseño, dispuestos para el uso de toda la comunidad de forma gratuita.

En los últimos 3 años de trabajo, la línea de ingeniería y diseño del nodo de Bogotá ha formado y acompañado el proceso de aprendizaje de cerca de 186 aprendices que 
generalmente tienen un perfil profesional como tecnólogos en diseño de productos industriales ó técnicos en mecánica industrial. Adicionalmente se prestaron servicios de asesoría a 70 empresas de todos los sectores productivos y se realizó el acompañamiento aproximadamente a 230 emprendedores, que buscaron la Red para materializar sus ideas de forma profesional. Durante estos casi tres años de ejecución del proyecto de la Red de Tecnoparque solamente el nodo Bogotá, se han asesorado 430 proyectos en total, se ha acompañado el proceso de solicitud de 15 patentes, se ha logrado realizar 45 articulaciones de proyectos conjuntos con universidades y grupos de investigación formales, inscritos al sistema nacional de ciencia y tecnología de Colombia - Colciencias y 74 empresas de diferentes sectores económicos.

Se estima que en toda la Red TecnoParque de Colombia, durante el año 2017 se asesoraron 1066 proyectos que culminaron en prototipos funcionales, 458 en prototipos puestos en el mercado, se articularon 171 grupos de investigación, se asesoraron 407 empresas y se realizaron 412 eventos de divulgación EDT. (SENNOVA SGPSV24 2018).

Este tipo de iniciativas ha propuesto una continuidad para estudiantes de formación técnica y tecnológica, que buscan continuar su formación de la mano de instructores, dinamizadores y profesionales especializados a partir de proyectos de base tecnológica y relacionamiento con industrias reales e instituciones universitarias.

Entre los factores de mejora que hemos encontrado para fortalecer y mejorar el proyecto de Red de Tecnoparques están:

-La divulgación de los servicios, resultados e infraestructura, que se tiene a disposición de toda la comunidad y sea más reconocida tanto a nivel local como nacional.

-Continuar con la mejora de la infraestructura y distribución de nodos por todo el territorio nacional, ya que la tecnología avanza con gran velocidad y es necesario estar a la vanguardia para prestar los servicios con herramientas de alta calidad y ser competitivos en términos de innovación.

-Promover estrategias y políticas de integración más fuertes con entidades públicas y privadas, para que más aprendices, emprendedores y empresas conozcan los servicios que ofrece Tecnoparque y puedan acceder a estos beneficios.

\section{Referencias bibliográficas:}

\section{Revista:}

Canchica de Medina, Marbelys Elizabeth. (2016). Modelo gavilán para el desarrollo de competencias en el manejo de información a través de google drive. una experiencia innovadora. Academia y Virtualidad, 9(2), 10-26. 10.18359/ravi.1822 


\section{Capítulo de un libro:}

Avila Forero, J. S. (2016). Design of training materials for teaching anatomy. En E. U. València (Ed.), IFDP'16 - Systems \& Design:Beyond Processes and Thinking (págs. 10151030). Valencia: Universitat Politècnica de València.

\section{Página web:}

Brown, T. (15 de 5 de 2016). http://dschool-old.stanford.edu. Obtenido de Design Thinking: http://dschool-old.stanford.edu/use-our-methods/

Historia, Visión, Misión, Valores y Símbolos. (2018). Lineabase.sena.edu.co. Retrieved 14 March 2018, from https://lineabase.sena.edu.co/acerca-del-sena/quienessomos/Paginas/Historia-Vision-Mision-Valores-y-Simbolos.aspx

SENA. (2018). Sena.edu.co. Retrieved 14 March 2018, from http://www.sena.edu.co/esco/formacion/Paginas/tecnologia-innovacion.aspx

SENNOVA SGPSV24. (2018). Sennova.senaedu.edu.co. Retrieved 13 January 2018, from http://sennova.senaedu.edu.co/

Somos. (2018). Sena.edu.co. Retrieved 14 March 2018, from http://www.sena.edu.co/es$\mathrm{co} /$ sena/Paginas/quienesSomos.aspx

Producto viable mínimo. (2017, 30 de octubre). Wikipedia, La enciclopedia libre. Fecha de consulta: 20:49, marzo 14, 2018 desde https://es.wikipedia.org/w/index.php?title=Producto_viable_m\%C3\%ADnimo\&oldid=1030 11339.

TecnoParque Colombia. (2018). Tecnoparque.sena.edu.co. Retrieved 14 March 2018, from http://tecnoparque.sena.edu.co/Paginas/default.aspx

Tecnología en Gestión de la Producción Industrial. (2018). Cdtisena.com. Retrieved 17

February 2018, from http://www.cdtisena.com/gestion-produccion-industrial.html 